\title{
A scoring system for histopathologic and immunohistochemical evaluations of uterine leiomyosarcomas
}

\author{
CHIKA YOSHIDA ${ }^{1}$, TOMOYUKI ICHIMURA ${ }^{1}$, NAOKI KAWAMURA ${ }^{2}$, AKEMI NAKANO $^{1}$, \\ MARI KASAI $^{1}$, TOSHIYUKI SUMI ${ }^{1}$ and OSAMU ISHIKO ${ }^{1}$ \\ ${ }^{1}$ Department of Obstetrics and Gynecology, Osaka City University Graduate School of Medicine, 1-4-3, \\ Asahimachi, Abeno-ku, Osaka 545-8585; ${ }^{2}$ Department of Obstetrics and Gynecology, Osaka City \\ General Hospital, 2-13-22, Miyakojimahondori, Miiyakojima, Osaka 534-0021, Japan
}

Received May 19, 2009; Accepted June 29, 2009

DOI: 10.3892/or_00000493

\begin{abstract}
Uterine leiomyosarcomas (LMS) are difficult to distinguish from benign leiomyomas without surgery. In this study we performed transcervical needle biopsy on 475 patients, 8 LMS patients and 467 patients with nonsarcomas (non-LMS) in a high-risk group for LMS, and evaluated whether examinations performed with Ki-67 and CD34 immunohistochemical analyses in addition to the standard hematoxylin-eosin (H\&E)-stained sections would improve preoperative diagnostic precision of the uterine smooth muscle tumors. Histopathologic analysis included three factors: degree of cytologic atypia, mitotic index and coagulative tumor cell necrosis $(\mathrm{CTCN})$. We also evaluated cell proliferation with Ki-67 expression. In cases of suspected CTCN, we examined CD34 expression and counted positive blood vessels in the necrotic area. Three of the 8 LMS cases satisfied the diagnostic criteria of LMS by histopathologic evaluation with H\&E-stained sections. We made a score list based on these analyses; scores for LMS specimens ranged from 6-14 points; non-LMS specimens scored 0-2 points. At the cut-off score of 6 points, the positive predictive value to distinguish LMS from non-LMS was $100 \%$, showing that this scoring system, is a useful method for preoperative differentiation between LMS and non-LMS tumors.
\end{abstract}

\section{Introduction}

Uterine leiomyomas are common benign smooth muscle tumors occurring in nearly $30 \%$ of women older than 30 years (1). Because of clinical introduction of gonadotropin releasing hormone agonists (2), and uterine arterial embolization (3),

Correspondence to: Dr Chika Yoshida, Department of Obstetrics and Gynecology, Osaka City University Graduate School of Medicine, 1-4-3 Asahimachi, Abeno-ku, Osaka 545-8585, Japan E-mail: c-yoshida@med.osaka-cu.ac.jp

Key words: leiomyosarcoma, needle biopsy, Ki-67, CD34, necrosis patients with uterine leiomyomas who might have previously undergone surgery, tend to be managed conservatively. In such situations, it is important to distinguish uterine leiomyosarcomas (LMS) from leiomyomas. Magnetic resonance imaging (MRI) has been one of the most useful examinations for preoperative differentiation between these tumors, but even with MRI, it is difficult to distinguish LMS from degenerating leiomyomas. Therefore, it is difficult to distinguish LMS from leiomyomas in some cases without histopathologic diagnosis using surgical specimens (4-6). The most reliable preoperative diagnostic method is biopsy of the tumor. Since 1994, we have performed transcervical needle biopsy, a procedure infrequently performed, to improve preoperative differential diagnosis of these tumors $(5,7-9)$. Uterine smooth muscle tumors, as proposed by Bell et al, are diagnosed histopathologically by three criteria: degree of cytologic atypia (CA), mitotic index (MI) and presence or absence of coagulative tumor cell necrosis (CTCN) (10).

However, such factors are not always included in the small specimens obtainable by needle biopsy, resulting in false negatives. Therefore, specimens obtained by needle biopsy might not be precisely diagnosed by morphologic evaluation of hematoxylin-eosin (H\&E)-stained sections alone; ancillary techniques may be necessary to improve diagnostic precision. In this study, we evaluated whether examinations using immunohistochemical analyses of Ki-67 and CD34 in addition to H\&E-stained sections would improve the diagnostic precision of needle biopsy specimens of uterine smooth muscle tumors.

\section{Materials and methods}

Patients. Between May 1994 and April 2008, 475 patients in a high-risk group for LMS underwent transcervical needle biopsies of their uterine myoma-like tumors to rule out malignancies. Our criteria for inclusion in this high-risk group of LMS were: a) myoma of unusual MRI findings (heterogeneous or diffuse high intensity on T1- and/or T2-weighted images), b) high serum lactate dehydrogenase concentrations $(>1.5 \times \mathrm{UNL}), \mathrm{c})$ rapidly growing tumors (an increase by 6 weeks' gestational size over one year), or d) growing tumors post-menopause $(11,12)$. All 475 patients (mean age \pm SD: 
$45.2 \pm 8.0$ years) gave written informed consent and the ethics committee of Osaka City University Hospital approved the study protocol.

Transcervical needle biopsy of uterine myoma-like tumors. The procedure used in transcervical needle biopsy of uterine myoma-like tumors was previously described (9). Biopsies were performed easily using a Pro-Mag 2.2 biopsy system (Manan Medical Products, Northbrook, IL) with an automatic cutting needle (25-cm long, 16-gauge, 17-mm notch) and a 20-cm long straight stainless-steel guide pipe (Honest Medical, Tokyo, Japan) with an external diameter of $4 \mathrm{~mm}$ and an internal diameter of $3 \mathrm{~mm}$. Before biopsy, we confirmed the location of the targeted myoma-like nodule, and the direction and distance from the uterine cavity using T2-weighted MRI images.

If two or more nodules were observed in the uterus, we chose the largest or most degenerate nodule. Patients were placed in the lithotomy position, and the guide pipe was inserted transcervically into the uterine cavity. Next, the operator inserted the guide pipe in the uterus and brought it to the targeted nodule under transabdominal ultrasonic guidance. The biopsy needle was then inserted through the guide pipe into the uterine muscle. After confirmation that the tip of the needle was within the nodule, the biopsy gun was fired and a core of tissue was obtained. To prevent sampling error, three or more biopsy cores per patient were obtained.

\section{Histopathologic analysis}

Cytologic atypia $(C A)$. The degrees of CA were recorded as none, mild or severe. To be considered severe, the atypia almost invariably had to be detectable at low magnification (x10 objective and x10 ocular), because significant atypia is usually manifested by nuclear pleomorphism.

Mitotic index (MI). Mitotic figures were counted in the most mitotically active area of the specimens. Mitotic figures were counted using a $\times 40$ objective and x10 ocular lens in all the fields. The count in the field that had the highest number of mitotic figures was recorded as the mitotic index of the tumor. We classified MI into a 3-point scale (0, 1-9, and $\geq 10 / \mathrm{HPF})$.

Pattern of necrosis. The pattern of the necrosis was mainly divided into CTCN and hyaline necrosis (HN). The characteristics of $\mathrm{HN}$ and $\mathrm{CTCN}$, as described by Bell et al (10) are shown Table I. CTCN, which was typical of LMS, was characterized by an abrupt transition from viable cells to the ghost cells, without an interposed zone of granulation or hyalinized tissue (Fig. 1A). HN, which was often found in leiomyomas, was observed as necrotic cells in the shape of hyalinized (Fig. 1D) or sometimes ghost cells (Fig. 1E). The band of hyalinized collagen was often present between the viable cells and the necrotic focus (Fig. 1C). In Fig. 1D, typical HN images are shown: HN1, consisting of hyalinized cells, and in Fig. 1E, other HN images: HN2, including ghost cells. When the interposed zone was not included in the small specimens by the needle biopsy, it was difficult to distinguish CTCN from HN2 (Fig. 1B and E). In this study, ghost cells in the necrotic focus without an interposed zone were recorded as 'suspected CTCN'. The 'suspected CTCN' group included cases of CTCN or HN2.

Two independent observers who were unaware of the clinical data determined histopathologic analyses based on the most advanced lesions in the specimens. Interobserver differences in the histopathologic diagnosis were resolved by a consensus of the two observers after re-evaluation.

Immunohistochemical analysis. Expression of Ki-67 and CD34 was investigated in paraffin-embedded sections using the avidin-biotin-peroxidase complex method. The paraffin sections ( $4 \mu \mathrm{m}$ thick) were de-paraffinized and immersed in $3 \%$ hydrogen peroxidase in methanol to block endogenous peroxidase activity. Next, an antigen retrieval procedure was performed by immersing the slides in $10 \mathrm{mM}$ citrate buffer ( $\mathrm{pH}$ 6.0) and heating in an autoclave at $120^{\circ} \mathrm{C}$ for $20 \mathrm{~min}$. The protocol for the Dako LSAB 2 Peroxidase Kit (Dako, Kyoto, Japan) was followed. The sections for Ki-67 were incubated overnight with the primary antibody in a humidity chamber at $4^{\circ} \mathrm{C}$. The sections for CD34 were incubated for 30 min with the primary antibody at room temperature. The primary antibodies used for this study were monoclonal mouse anti-human Ki-67 and CD34 antibodies (Dako). The working dilutions of the primary antibodies were: 1:20 for anti-Ki-67, 1:40 for anti-CD34. The sections were rinsed with PBS for $15 \mathrm{~min}$ and incubated for $10 \mathrm{~min}$ with the secondary antibody (biotinylated goat anti-mouse and rabbit immunoglobulin G secondary antibody; Dako). The sections were then incubated with streptavidin-peroxidase complex using 3,3'-diaminobenzidine as a chromogen. Counterstaining was performed with Mayer's hematoxylin. The specificity of the immunohistochemical reactions was checked by omitting the primary antibody.

Counting procedure for immunohistochemistry. Cells were considered positive for Ki-67 expression when the nuclei were stained brown (Fig. 2A and B). In this study, we evaluated Ki-67 expression using the Ki-67 labeling index (LI) at high magnification (x40 objective and x10 ocular). We counted Ki-67-stained nuclei in the all fields in each specimen, and the highest LI was recorded. Viable blood vessel endothelia were stained brown by CD34. We defined positive blood vessels as those stained brown by CD34 in the necrotic area (Fig. 2C and D). We counted CD34-stained vessels in all fields in each specimen at high magnification (x40 objective and x10 ocular), and the highest number of positive vessels was recorded. Expression of Ki-67 and CD34 was classified with a 3-point scale (negative, suspected positive and positive) based on results of the counts.

Scoring system. We evaluated the 3 factors of H\&E-stained sections (CA, MI and CTCN) and Ki-67 expression on a 3point scale for all cases. When suspected CTCN figures were observed in the specimens, we added expression of CD34. We defined the scores of CA as: negative, 0 points; mild 1 point and severe 3 points. We defined the scores for MI as: 0 mitotic figures, 0 points; $1-9$ mitotic figures, 1 point; $\geq 10$ mitotic figures, 3 points. The points for CTCN were: absence, 0 points; suspected CTCN, 1 point; frank presence of CTCN, 3 points. In this system, we set 6 points as the cut- 

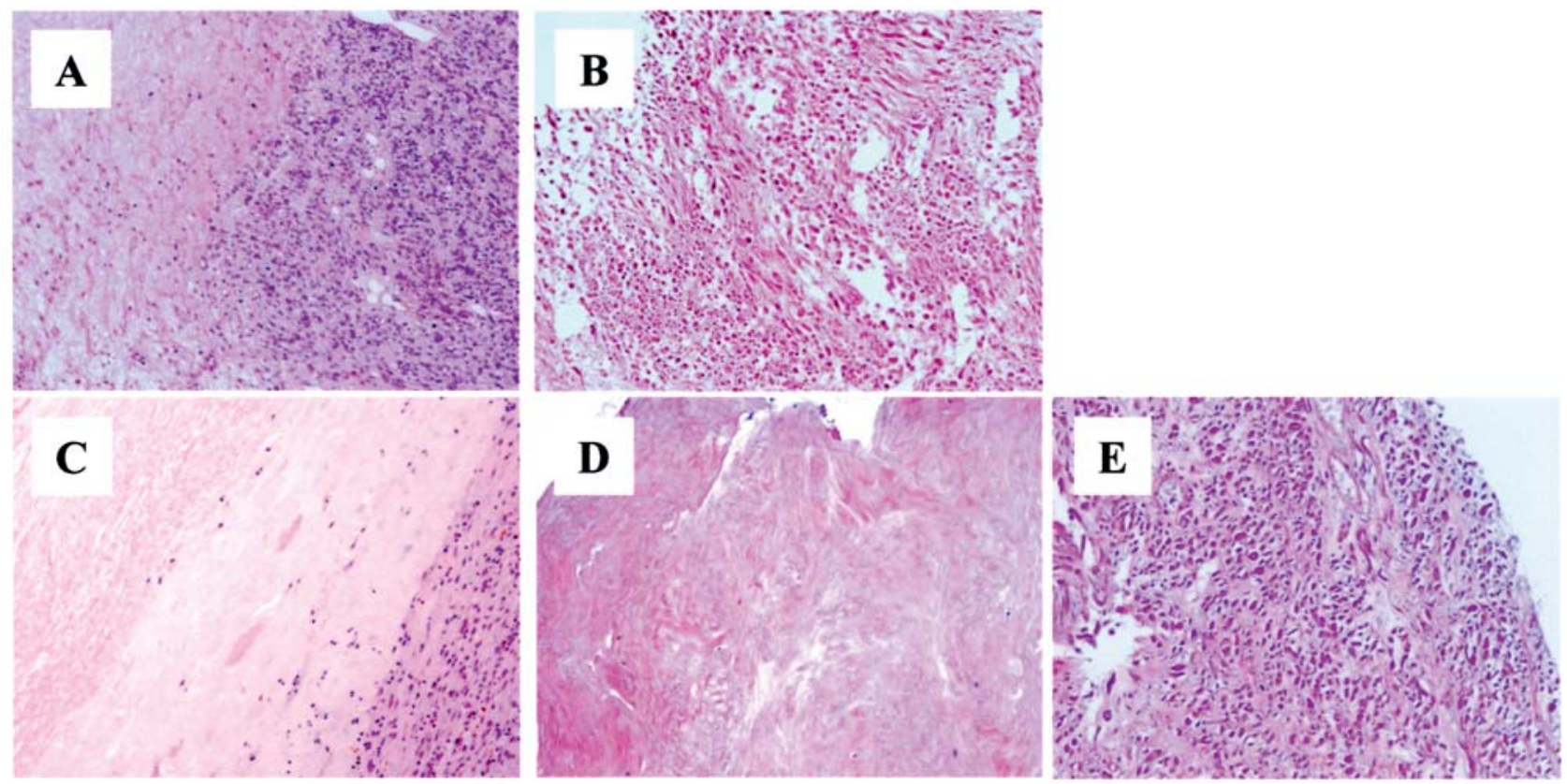

Figure 1. (A and B) CTCN in LMS. (A) CTCN was characterized by an abrupt transition from viable cells to ghost cells without an interposed zone of granulation tissue or hyalinized tissue between viable and necrotic cells. (C-E) HN in leiomyoma. (C) Between the viable cells and the necrotic focus, the band of paucicellular hyalinized collagen of varying thickness was present. (D; HN-1) Typical hyaline necrosis, consisting of hyalinized cells. (E; HN-2) Hyaline necrosis, consisting of ghost cells.
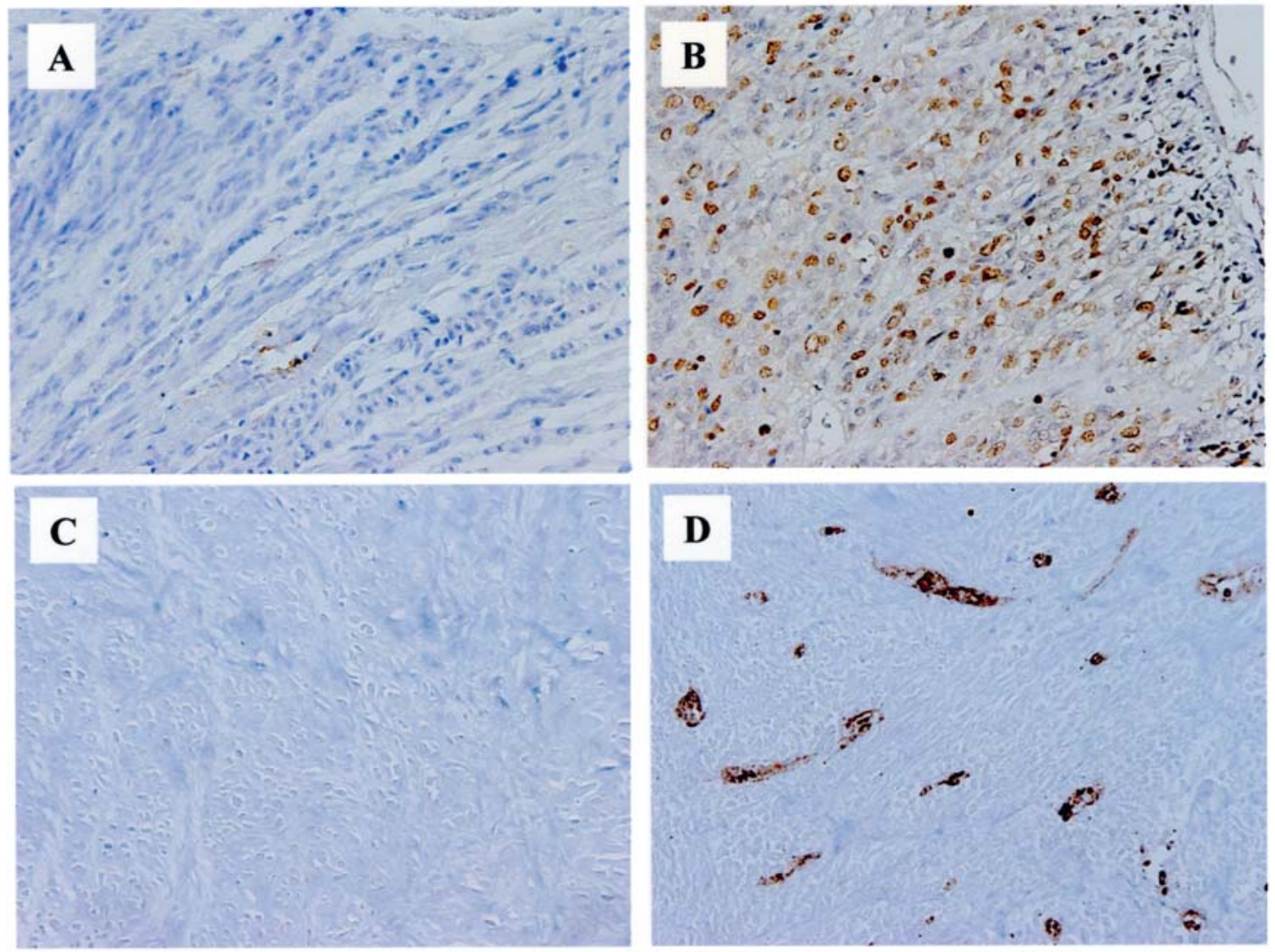

Figure 2. Immunohistochemical staining of Ki-67 and CD34. (A) Ki-67 expression in non-LMS. (B) Ki-67 expression in LMS. (C) CD34 expression in nonLMS. (D) CD34 expression in LMS.

off score for the diagnosis of LMS. Ki-67 and CD34 expressions were evaluated on a 3-point scale: negative, 0 points; suspected positive, 1 point; positive, 5 points.
Statistical analysis. A conventional relative operating characteristic (ROC) curve was used to determine the cut-off score that yielded the highest combined sensitivity and 

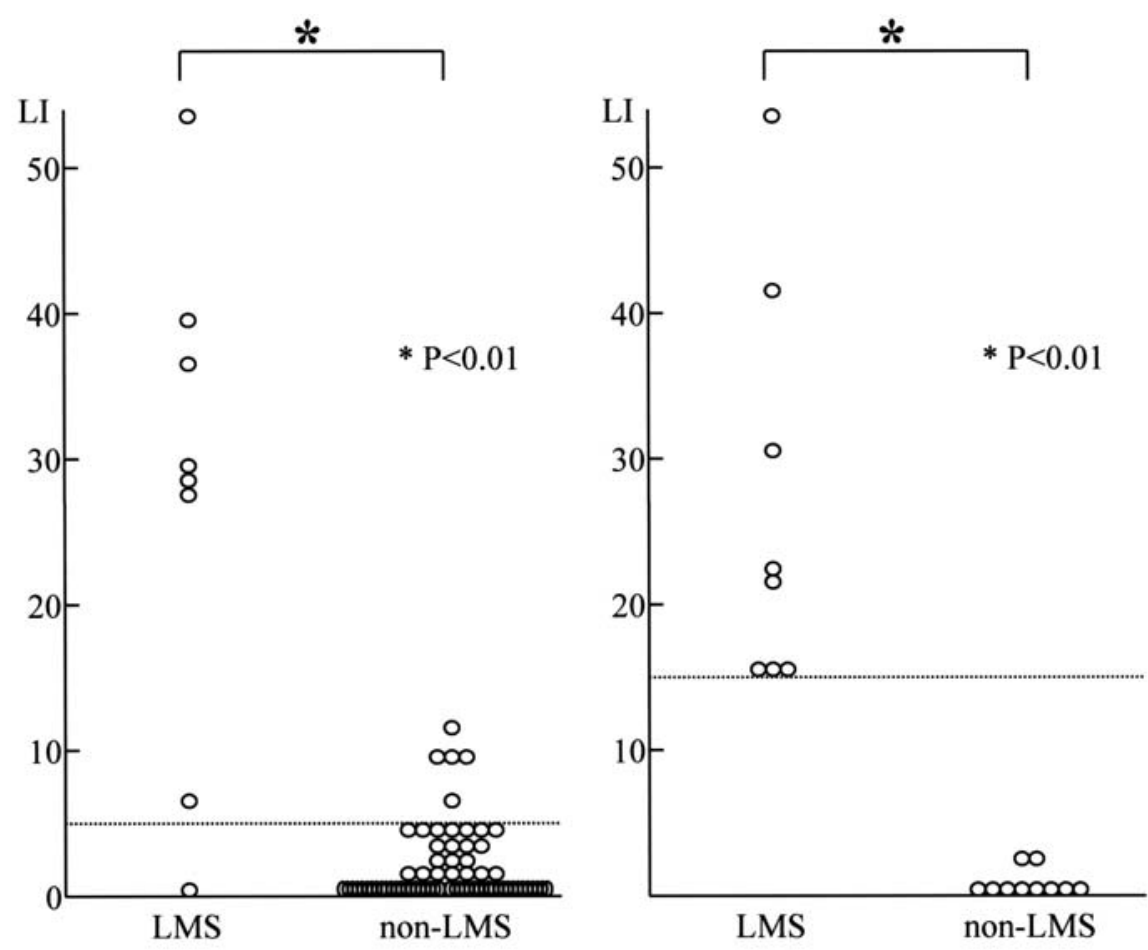

Figure 3. (A) Ki-67 expression in LMS and non-LMS biopsy specimens. The dotted line was the cut-off line (5\%). (B) Ki-67 expression in LMS and non-LMS surgical specimens. The average value of LMS was $26.5 \pm 14.0$, and that of non-LMS was $0.4 \pm 0.8$. The dotted line was the cut-off $(15 \%)$. ${ }^{*} \mathrm{P}<0.01$ (MannWhitney U test).

specificity with respect to distinguishing LMS from nonLMS. Confidence intervals of 95\% (95\% CI) for sensitivity, specificity, positive predictive value and false negative rate were calculated using standard $\mathrm{Z}$ tests. The Mann-Whitney $\mathrm{U}$ test was used to analyze the data for significant differences. Differences were considered statistically significant at $\mathrm{P}<0.01$. All data were expressed as means $\pm \mathrm{SD}$.

\section{Results}

Among 475 patients, 8 cases satisfied the diagnostic criteria of LMS with surgical or biopsy specimens. The results of H\&E-stained sections are shown in Table II. For diagnoses of LMS with regard to CA, mild atypia was used as the cutoff line, which gave the following values for the test: sensitivity, $75.0 \%$ (95\% CI, 44.6-96.8\%); specificity, $94.4 \%$ (95\% CI, 92.2-96.3\%); positive predictive value, $18.8 \%$ (95\% CI, 7.5-36.4\%); false negative rate, $25.0 \%$ (95\% CI, 3.7-65.1\%). The cut-off line for MI was 1/HPF, which gave the following values: sensitivity, $50.0 \%$ (95\% CI, 18.9$84.3 \%$ ); specificity, $92.3 \%$ (95\% CI, 89.7-94.5\%); positive predictive value, $10.0 \%$ (95\% CI, 2.9-23.7\%); false negative rate, $50.0 \%$ (95\% CI, 18.9-84.3\%). For necrosis patterns, using 'suspected CTCN' as the cut-off line gave the following values: sensitivity, $75.0 \%$ (95\% CI, 44.6-96.8\%); specificity, $94.2 \%$ (95\% CI, 92.0-96.1\%); positive predictive value, $18.2 \%$ (95\% CI $7.2-35.4 \%$ ); false negative rate, $25.0 \%$ (95\% CI, 3.7-65.1\%). These results show little difference in diagnostic precision among the three factors.

The results of LI of Ki-67 are shown in Fig. 3A. For the LMS group, Ki-67 expression gave values of 0-53\%, with an average value of $27.3 \pm 17.2$. One LMS case showed $0 \% \mathrm{Ki}-67$
Table I. Pattern of necrosis.

\begin{tabular}{|c|c|c|c|}
\hline & $\begin{array}{c}\text { Border between } \\
\text { viable and ghost } \\
\text { cells }\end{array}$ & $\begin{array}{c}\text { Ghost cells in } \\
\text { necrosis }\end{array}$ & $\begin{array}{l}\text { Blood vessel } \\
\text { in necrosis }\end{array}$ \\
\hline CTCN & $\begin{array}{l}\text { Clear } \\
\text { Band of } \\
\text { hyalinized } \\
\text { collagen (-) }\end{array}$ & $\begin{array}{l}\text { Preserved } \\
\text { pleomorphism } \\
\text { of nuclei }\end{array}$ & $\begin{array}{l}\text { Preserved } \\
\text { pre-existing } \\
\text { vascular }\end{array}$ \\
\hline $\mathrm{HN}$ & $\begin{array}{l}\text { Band of } \\
\text { hyalinised } \\
\text { collagen }(+)\end{array}$ & $\begin{array}{l}\text { Sometimes } \\
\text { observed } \\
\text { (generally observed } \\
\text { hyalinised cells) }\end{array}$ & $\begin{array}{l}\text { Pre-existing } \\
\text { vascular has } \\
\text { pale ghost } \\
\text { outline }\end{array}$ \\
\hline
\end{tabular}

expression because most of the specimen included CTCN. In the non-LMS group, 441 cases showed no expression of Ki-67. However, 26 of the 467 non-LMS cases showed Ki-67 LI greater than $1 \%$. In the highest case, the LI reached $11 \%$, while the average value was $0.2 \pm 1.1$.

According to ROC curve analysis, $5 \%$ was the cut-off value that achieved the highest combined sensitivity and specificity with respect to distinguishing LMS from nonLMS. At this value, sensitivity and specificity were $87.5 \%$ (95\% CI, 64.3-99.7\%) and 98.9\% (95\% CI, 97.9-99.7\%), respectively. In addition, when we evaluated similar examination with $8 \mathrm{LMS}$ and 10 non-LMS surgical specimens, the Ki-67 LI for LMS reached $\geq 15 \%$ in all cases (Fig. 3B). Based 


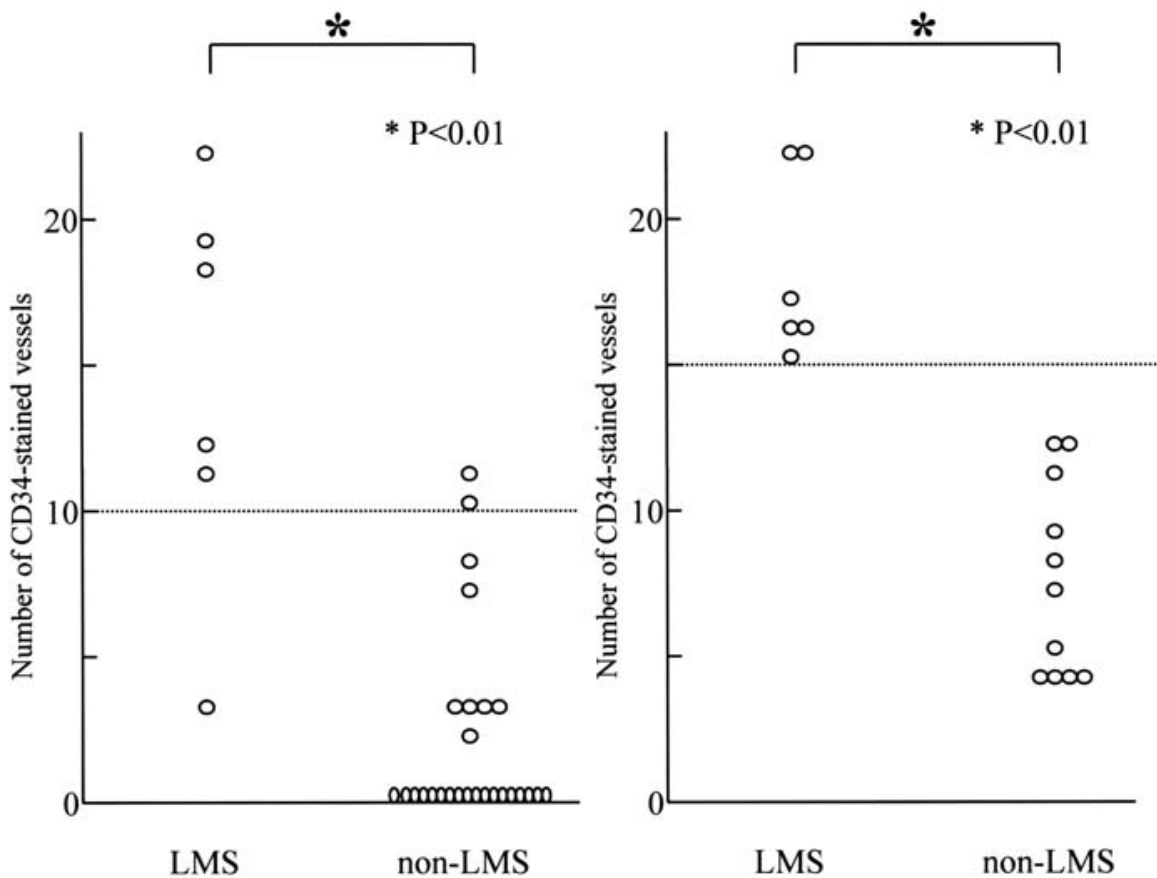

Figure 4. (A) CD34 expression in LMS and non-LMS biopsy specimens. The dotted line was the cut-off (10 CD34-stained vessels). (B) CD34 expression

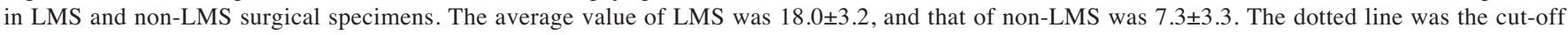
(15 CD34-stained vessels). ${ }^{*} \mathrm{P}<0.01$ (Mann-Whitney U test).
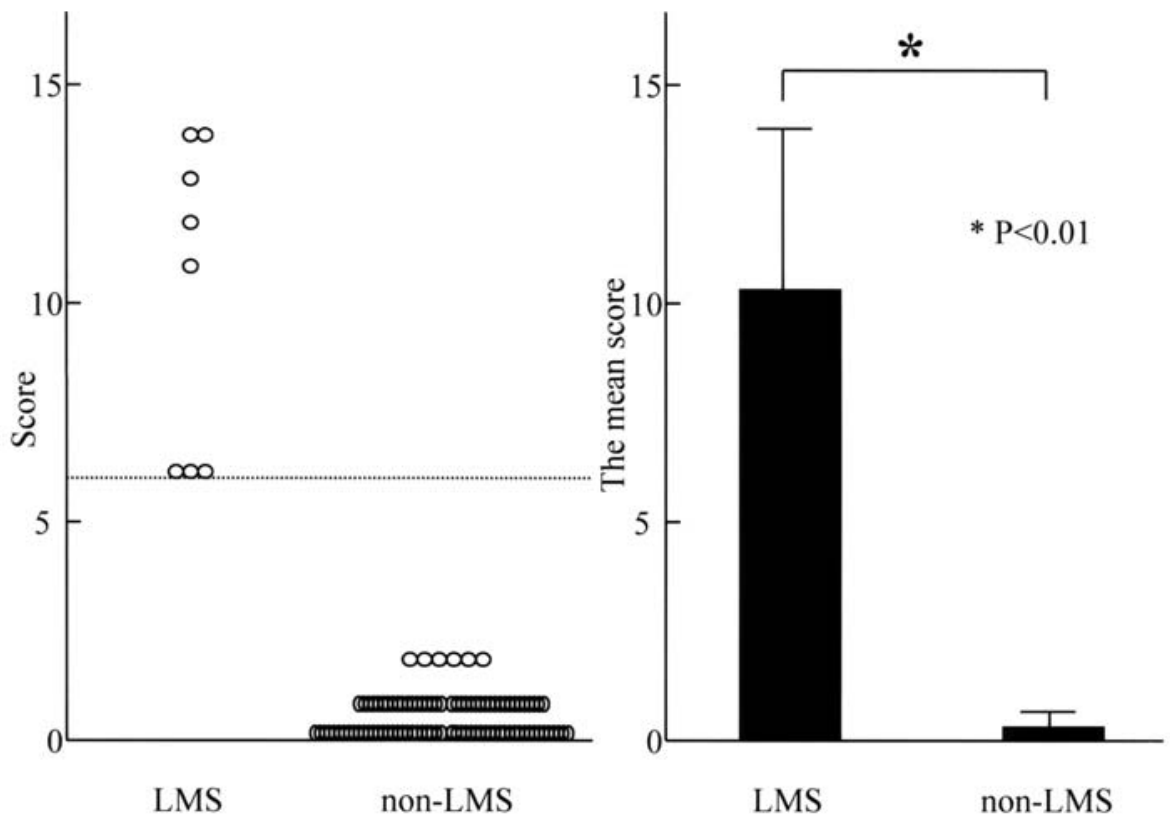

Figure 5. (A) The total score of histopathologic and immunohistochemical analysis in LMS group and the non-LMS group. The dotted line was the cut-off line (6 points). (B) The mean score of LMS group and non-LMS group. The mean score of LMS group was significantly higher than that of non-LMS group. Bars represent the SD. $\mathrm{P}<0.01$ (Mann-Whitney U test).

on these results, cases with $\mathrm{Ki}-67 \mathrm{LI}>5 \%$ were defined as 'suspected positive' and cases with $\mathrm{Ki}-67 \mathrm{LI} \geq 15 \%$ were defined as positive.

The results of counts of the CD34-stained vessels are shown in Fig. 4A. Thirty-three cases, 6 LMS and 27 nonLMS, had 'suspected CTCN', including HN2 or CTCN, in specimens obtained by needle biopsy. Among the 6 LMS cases, the CD34-stained vessels in these specimens ranged from 3 to 22 ; the mean was $14.2 \pm 6.9$. Among the 27 nonLMS cases, 18 were negative for $\mathrm{CD} 34$. Of the remaining 9 cases, one had 11 positive blood vessels; the mean value was $1.9 \pm 3.3$. According to ROC curve analysis, the optimal cut-off value was $10 \mathrm{CD} 34$-stained vessels, which gave sensitivity and specificity values of $83.3 \%$ (95\% CI, 52.8$99.6 \%$ ) and $96.3 \%$ (95\% CI, 89.5-99.9\%), respectively. As with the Ki-67 expression, 17 surgical specimens, including 
Table II. The histopathologic factors and relations with the LMS group and the non-LMS group.

\begin{tabular}{lrc}
\hline & Non-LMS & LMS \\
\hline CA & & \\
None & 441 & 2 \\
Mild & 26 & 2 \\
Severe & 0 & 4 \\
Total & 467 & 8 \\
MI & & \\
0 & 431 & 4 \\
$1-9$ & 36 & 2 \\
$\geq 10$ & 0 & 2 \\
Total & 467 & 8 \\
CTCN & & \\
None & 440 & 2 \\
Suspected & 27 & 3 \\
Positive & 0 & 3 \\
Total & 467 & 8 \\
\hline
\end{tabular}

6 LMS and 11 non-LMS, were immunostained for CD34. All LMS specimens had $\geq 15$ CD34-stained vessels (Fig. 4B). Therefore, we defined biopsy samples that had $>10$ CD34stained vessels as 'suspected positive' and those with $\geq 15$ CD34-stained vessels as positive.

The total scores of histopathologic and immunohistochemical analyses for both the LMS group and the nonLMS group are shown in Fig. 5A. When we diagnosed the needle biopsy specimens of 475 cases with this scoring system in a retrospective study, the total scores of all 8 LMS cases satisfied our diagnostic criteria for LMS by 6 points or more; the mean value was $10.3 \pm 3.7$. In contrast, all 467 nonLMS cases scored under 6 points using the same criteria; the mean value was $0.2 \pm 0.4$. In addition, the mean scores of the LMS group were significantly higher than the mean score of the non-LMS group (Fig. 5B).

\section{Discussion}

The purpose of this study was to establish a method to augment H\&E-stained sections with tests for $\mathrm{Ki}-67$ and CD34, thereby improving preoperative differential diagnosis between LMS and leiomyomas. LMS in the surgical specimens is usually diagnosed by morphologic evaluation with H\&Estained sections. Specimens with at least two of three indicators (severe atypia, $\geq 10$ mitoses/HPF and/or presence of CTCN) were diagnosed as LMS (10). In this study the three factors used in H\&E-stained biopsy specimens showed high specificity. Therefore, histopathologic evaluations with H\&E stains were useful for screening out non-LMS specimens taken from the high-risk group for LMS. But we thought that it was difficult to diagnose LMS precisely with only these factors because sensitivity and positive predictive values were low. Ki-67 antigen is a nuclear protein expressed in all active phases of the cell cycle from late $S$ phase forward, and is especially abundant in the $G_{2}$ and $M$ phases; however, it is absent in $\mathrm{G}_{0}$ (13). Because $\mathrm{Ki}-67$ is regarded as an indicator of cell proliferative activity, and by extension, biological aggressiveness (14), it is unsurprising that $\mathrm{Ki}-67$ is overexpressed in LMS cases, compared with non-LMS cases. Several studies have reported that expression of Ki-67 was useful for differentiating between LMS and non-LMS in surgical specimens; these results showed high precision $(13,15,16)$.

Unfortunately, the use of Ki-67 may give false positives. In this study, the cut-off score that achieved the highest combined sensitivity and specificity in biopsy specimens was $5 \%$. However, the LI of 5 non-LMS specimens were $>5 \%$; of these, 2 cases were atypical leiomyomas (AL). Other studies also described cases of cellular leiomyomas, AL or uterine smooth muscle tumors of uncertain malignant potential with Ki-67 LI >5\% (16-18). When we performed Ki-67 expression of 8 LMS and 10 non-LMS surgical specimens, all LMS samples had $\mathrm{Ki}-67 \mathrm{LI} \geq 15 \%$. We raised the cut-off score for positive diagnosis in the biopsy samples to $15 \%$ based on this finding, and redefined 'suspected positive' as having a Ki-67 $\mathrm{LI}>5 \%$.

The Ki-67 expression also gave a false negative in one extremely necrotic LMS specimen in this study. Therefore, we thought it necessary to evaluate necrotic biopsy specimens closely to improve diagnostic precision. $\mathrm{CTCN}$ is an important finding in histopathologic evaluations for LMS (10). Because specimens can be obtained from various areas by the surgery to perform histopathologic evaluation, there is little need that the surgical specimens show only CTCN. Therefore, there has been no study on the evaluation of the blood vessels in the necrotic area included in the uterine smooth muscle tumors. In this study, we directed our attention to the CD34-stained vessels in the necrotic areas and ascertained whether we could distinguish between HN2 and CTCN. Five of the 6 LMS biopsy cases had $>10$ CD34-stained vessels in the necrotic areas; the highest combined sensitivity and specificity was provided when the cut-off score was set to 10. However, in 2 of 27 non-LMS cases, we found $\geq 10$ positive blood vessels. When we performed the expression for CD34 in the necrotic surgical specimens, $\geq 15$ positive blood vessels were observed in each LMS specimen. From these results, we defined cases with $\geq 10$ positive vessels as 'suspected positive' and cases with $\geq 15$ positive blood vessels as positive. Thus, the immunohistochemical factors were superior in sensitivity and specificity to H\&E-stained sections. However, it was difficult to diagnose LMS accurately using only the expressions for Ki-67 and CD34. Therefore, we made a scoresheet based on the histopathologic and immunohistochemical analyses, with the total scores before surgery, to distinguish between LMS and non-LMS specimens. Bell et al proposed, in regard to the diagnosis of LMS using H\&E-stained surgical sections, that cases which satisfied at least 2 of the 3 criteria (severe atypia, 10 mitoses/ HPF, presence of CTCN) be diagnosed as LMS; we used this paradigm to diagnose LMS in the biopsy specimens in this study (10).

Each biopsy specimen received 3 points for each positive criterion. Cases having two or more positive factors (i.e., with 6 points or more) were diagnosed as LMS. They received 
1 point for each 'suspected positive' criterion, which prevented LMS diagnoses when a case received one positive and two 'suspected positive' criteria.

When we set the scores for CD34 and Ki-67, two elements were considered. The first element was that the diagnostic precision of Ki-67 and CD34 expression compared well with the H\&E-stained sections. The second element was that we should be able to diagnose LMS by adding immunohistochemical factors when the biopsy specimens had only one factor among the afore-mentioned three factors of H\&Estained sections. For example, if the biopsy specimens had only necrotic area, we gave the case 1 point as 'suspected CTCN' in H\&E-stained section. However, if a case had $\geq 15$ CD34-stained vessels in the necrotic area, there seemed little doubt that the case was LMS. Therefore, we added 5 points to cases showing positive Ki-67 or CD34 expression because those cases should be diagnosed as LMS, with 6 points.

In conclusion, we show that our scoring system with histopathologic and immunohistochemical assays is a useful method to distinguish between LMS and non-LMS in small specimens obtained by needle biopsy. However, in this study we evaluated the cases and set the scores in a retrospective study. It is therefore necessary to evaluate whether this new scoring system is useful in a prospective study.

\section{Acknowledgements}

We would like to thank all of the gynecologists at Osaka City University Graduate School of Medicine and Osaka City General Hospital for their support.

\section{References}

1. Vollenhoven BJ, Lawrence AS and Healy DL: Uterine fibroids: a clinical review. Br J Obstet Gynaecol 97: 285-298, 1990.

2. Adamson GD: Treatment of uterine fibroids: current findings with gonadotropin-releasing hormone agonists. Am J Obstet Gynecol 166: 746-751, 1992.

3. McLucas B, Adler L and Perrella R: Uterine fibroid embolization: nonsurgical treatment for symptomatic fibroids. J Am Coll Surg 192: 95-105, 2001

4. Kawamura N, Iwanaga N, Hada S, et al: Transient shrinkage of a uterine leiomyosarcoma treated with $\mathrm{GnRH}$ agonist for a presumed uterine leiomyoma: comparison of magnetic resonance imaging finding before and during $\mathrm{GnRH}$ agonist treatment. Oncol Rep 8: 1255-1257, 2001.
5. Kawamura N, Ito F, Ichimura T, et al: Transient rapid growth of uterine leiomyoma in a postmenopausal woman. Oncol Rep 6: 1289-1292, 1999.

6. Schwartz LB, Diamond MP and Schwartz PE: Leiomyosarcomas: clinical presentation. Am J Obstet Gynecol 168: 180-183, 1993.

7. Kawamura N, Shibata S, Ito F, Ichimura T, Umesaki N and Ogita S: Practical method for transcervical needle biopsy of uterine leiomyomas to avoid unnecessary surgery. J Gynecol Tech 2: 201-206, 1996.

8. Kawamura N, Ichimura T, Ito F, et al: Transcervical needle biopsy for the differential diagnosis between uterine sarcoma and leiomyoma. Cancer 94: 1713-1720, 2002.

9. Kawamura N, Ichimura T, Takahashi K, Tujimura A, Ishiko O and Ogita S: Transcervical needle biopsy of uterine myoma-like tumor using an automatic biopsy gun. Fertil Steril 77: 1060-1064, 2002.

10. Bell SW, Kempson RL and Hendrickson MR: Problematic uterine smooth muscle neoplasms. A clinicopathologic study of 213 cases. Am J Surg Pathol 18: 535-558, 1994.

11. Parker WH, Fu YS and Berek JS: Uterine sarcoma in patients operated on for presumed leiomyoma and rapidly growing leiomyoma. Obstet Gynecol 83: 414-418, 1994.

12. Buttram VC Jr and Reiter RC: Uterine leiomyomata: etiology, symptomatology and management. Fertil Steril 36: 433-445, 1981.

13. Cattoretti G, Becker MH, Key G, et al: Monoclonal antibodies against recombinant parts of the Ki-67 antigen (MIB1 and MIB3) detect proliferating cells in microwave-processed formalin-fixed paraffin sections. J Pathol 168: 357-363, 1992.

14. Layfield LJ, Liu K, Dodge R and Barsky SH: Uterine smooth muscle tumors: utility of classification by proliferation, ploidy, and prognostic markers versus traditional histopathology. Arch Pathol Lab Med 124: 221-227, 2000.

15. Mayerhofer K, Lozanov P, Bodner K, Bodner-Adler B, Kimberger $\mathrm{O}$ and Czerwenka $\mathrm{K}$ : $\mathrm{Ki}-67$ expression in patients with uterine leiomyomas, uterine smooth muscle tumors of uncertain malignant potential (STUMP) and uterine leiomyosarcomas (LMS). Acta Obstet Gynecol Scand 83: 1085-1088, 2004.

16. Mittal K and Demopoulos RI: MIB-1 (Ki-67), p53, estrogen receptor, and progesterone receptor expression in uterine smooth muscle tumors. Hum Pathol 32: 984-987, 2001.

17. Chen L and Yang B: Immunohistochemical analysis of P16, P53 and Ki-67 expression in uterine smooth muscle tumors. Int J Gynecol Pathol 27: 326-332, 2008.

18. Cramer SF, Newcomb PM and Bonfiglio TA: Myometrial dysplasia (atypical myometrial hyperplasia). Hum Pathol 38: 652-655, 2007. 